\title{
Checklist of vascular plants from Batu Caves, Selangor, Malaysia
}

\author{
Ruth Kiew \\ The Herbarium, Forest Research Institute Malaysia, 52109 Kepong, Selangor, Malaysia \\ E-mail: ruth@frim.gov.my
}

\begin{abstract}
The vascular plant flora of Batu Caves, a tower karst limestone formation, includes 269 species; 51 species (19\%) are Peninsular Malaysian endemics and 80 species (30\%) are calciphiles of which 56 (21\%) are obligate calciphiles and 26 species are obligate calciphiles endemic to Peninsular Malaysia. Four taxa are endemic to Batu Caves itself. That Batu Caves harbours a sizeable fraction (21.4\%) of Peninsular Malaysia's limestone flora underlines the need for detailed checklists of each and every limestone hill to enable adequate planning of conservation programmes to support biodiversity. Because botanical collecting began in the 1890s, Batu Caves is important as the type locality of 24 plant species. Land-use pressures have over time eliminated the surrounding native vegetation, leaving the flora vulnerable to aggressive weedy and alien species. Although designated as a Public Recreation Area, its protection status needs to be enforced and the boundaries clearly marked.
\end{abstract}

DOI: $10.15560 / 10.6 .1420$

\section{INTRODUCTION}

Batu Caves $\left(3^{\circ} 14^{\prime} \mathrm{N}, 101^{\circ} 41^{\prime} \mathrm{E}\right)$, or Gua Batu (in Malay), is a limestone tower karst formation $11 \mathrm{~km}$ northeast of the capital Kuala Lumpur. It rises to $329 \mathrm{~m}$ tall and covers about $2.59 \mathrm{~km}^{2}$. This massif with its vertical cliffs and craggy summit is a dominant landscape feature. Batu Caves is most famous for the Sri Subramaniaswamy Temple that at the Thaipusam festival is visited by hundreds of thousands of devotees who climb the 277 steps up to the Temple Cave. Besides its cultural importance as a religious site, it is also important for its cave ecosystems and associated fauna (Moseley et al. 2012) and for its flora (Wycherley 1972).

Batu Caves has been exploited commercially for a very long time. At first by Chinese farmers who since the $1860 \mathrm{~s}$ collected guano from the caves (Yussof 1997). Quarrying for limestone had already started by 1889 when H.N. Ridley first investigated the caves, flora and fauna. Although Batu Caves was designated as a Public Recreational Area in 1930, quarry licenses continued to be leased in spite of lobbying for the total protection of the massif by the Batu Caves Protection Association and the Malayan Nature Society. Only when quarrying caused rock falls in the Dark Cave adjacent to the Temple Cave did quarrying finally stop in 1981. Batu Caves is now surrounded by residential, light industry and temple buildings with very little if any of the original vegetation that surrounded the foot of the tower karst. Enforcement of its status as a Public Recreational Area is lacking and its boundaries are not clearly marked so that intrusions go unchecked.

The limestone flora in Malaysia is botanically important due to (a) its species richness-14\% of Peninsular Malaysia's species occur on the $0.04 \%$ of land area that limestone covers (Chin 1977), the result of the many different microhabitats stacked on a single limestone hill (Kiew 1991); (b) it is distinctly different in species composition compared with other forest types both in its common species, for example, species of Dipterocarpaceae, the dominant tree family in Malaysian rain forest, are hardly represented on limestone, and in calciphile species that are restricted to growing on limestone substrate, and (c) in its high level of endemism-21.4\% of species (Chin 1977). Saw et al. (2009) considered that the limestone flora is one of the most endangered vegetation types in Peninsular Malaysia because of its lack of legal protection and threats from quarrying and disturbance resulting from land use changes.

H.J. Kelsall was the first collector of plants from Batu Caves when in 1891 he discovered three new species (Adenoncos parviflora, Paraboea paniculata and P. verticillata). Ridley has made the most comprehensive collections when he visited in June 1889, December 1896, July and August 1897, August 1908 and December 1920 and described many new species (Wycherley 1972). Then it was apparently easier to access the summit of Batu Caves, but since this time quarrying has left sheer rock faces where previously there were accessible gullies leading to the summit. Now the collector is faced by precipitous rock faces. Ridley collected at a time when Batu Caves was still surrounded by pristine lowland forest, although coffee plantations and later rubber tree plantations were expanding towards Batu Caves. This forest has long since been completely cleared causing the first recorded extinction of a plant species, Echinodorus ridleyi Steenis (Alismataceae), in Malaysia. To date, due to its proximity to Kuala Lumpur, more than 35 botanists have sporadically collected specimens from Batu Caves and their research continues to add new records. In fact, Batu Caves is probably the best collected tower karst hills in Peninsular Malaysia. Wycherley (1972) provided a partial list of 199 vascular plant species collected by Ridley.

The aim of this updated checklist is to consolidate what is known of the flora of Batu Caves both from the 
many diverse literature sources as well as from herbarium specimens. This is necessary as a basis for drawing up conservation management programmes as well as for tracking the decline or loss of species and the invasion of aggressive alien or weedy species.

\section{MATERIALS AND Methods}

The checklist is based on a search of the literature (Henderson 1939, Wycherley 1972, and Chin 1977, $1979,1983 a, b$ are major works, but there are also many specialist articles on specific species) and herbaria that house major collections of specimens collected from Batu Caves, namely The Singapore Herbarium, Singapore Botanic Gardens, Singapore (SING); the Kepong Herbarium, Forest Research Institute Malaysia, Kepong, Selangor (KEP); and the University of Malaya Herbarium, Kuala Lumpur (KLU). The collections at KEP and SING are partially databased using BRAHMS (Botanical Research and Herbarium Management System), which greatly facilitated extraction of data. Extraction of data from KLU was done manually from accession books. In the case of dubious identifications, the herbarium specimen was checked and the name corrected. Only species recorded from the tower karst or limestone-derived soil at the base are included. Thus forest species that Ridley collected are not included in the checklist, nor are weeds that grow on wasteland around Batu Caves, nor exotic aliens that have invaded disturbed areas at the foot of Batu Caves.

The checklist includes family and species names, cites specimens, provides the endemic status, whether endemic in Peninsular Malaysia (E) or endemic in the phytogeographic zone that straddles the border of Peninsular Malaysia and Peninsular Thailand (ET); and status as a calciphile, i.e. whether it is an obligate calciphile restricted to growing on limestone (R) or whether it is a characteristic species most usually found on limestone (U).

\section{RESULTS}

The checklist (Appendix 1) includes 5 lycophyte species, 27 ferns, 2 gymnosperms, 182 dicotyledons and 53 monocotyledons, in total 269 species. This represents $22 \%$ of 1,216 species recorded growing on limestone in Peninsular Malaysia (Chin 1977). The percentages of endemic and obligate and characteristic calciphiles (Table 1) are representative of the limestone flora as a whole.

The ten most common families are Orchidaceae (23 species), Apocynaceae (20 species), Rubiaceae (14 species), Araceae (12 species) and Gesneriaceae, Moraceae and Urticaceae (each represented by 9 species). Ficus (Moraceae) with 9 species is by far the largest genus.

Of the 56 species of obligate calciphiles (Appendix 1), 26 are endemic in Peninsular Malaysia and are therefore of highest conservation concern. Among these are:

- 4 local endemic species or varieties that are obligate calciphiles and are only known from Batu Caves-
Epithema parvibracteatum, Pararuellia sumatrensis var. ridleyi, Pseuderanthemum lilacinum and Rhaphidophora burkillana

- 3 local endemic species that are obligate calciphiles and are only known from Batu Caves and the nearby Bukit Takun and Bukit Anak Takun that lie about $10 \mathrm{~km}$ north of Batu Caves-Maxburretia rupicola, Ophiorrhiza fruticosa and Paraboea paniculata

- very rare species found on one or two other limestone hills, e.g., Impatiens ridleyi is known only from Batu Caves and from Gunung Senyum, Pahang.

Other rare species that are not obligate calciphiles but are of conservation importance are

- 4 narrowly endemic species confined to an area within $15 \mathrm{~km}$ from Batu Caves-Begonia phoeniogramma, Beaumontia murdochii, Piper argyrites and Psychotria lanceolaria

- 6 widespread species that in Peninsular Malaysia are known only from Batu Caves-Piper mucronata, Pomatocalpa andamanica, Sageretia thea, Sapium insigne, Sauropus macranthus, and Trigonostemon villosus.

Batu Caves is also important because it is the type locality for 24 taxa, even though some have since been reduced to synonymy.

\section{Discussion}

Table 1 illustrates the fact that while the limestone flora is species rich $(1,216$ species), only a fraction are found on a single hill (21.4\% of the species on Batu Caves) due in part to local endemism of the obligate calciphiles. This is illustrated by the Gesneriaceae. For the Peninsular limestone flora as a whole, Gesneriaceae is ranked fourth with 39 species but on Batu Caves it is represented by just seven species. Of these, only two grow on nonlimestone substrates and are widely distributed; the rest are obligate calciphiles, two are local endemics, while the remaining three are more widespread but none are found on every hill. In fact it is the exception for limestone species to be encountered on every or even most hills. This is especially true among the obligate calciphiles. For instance, eight species of balsam are obligate calciphiles but only Impatiens ridleyi grows on Batu Caves and one other hill (Gunung Senyum, Pahang). The implications for conservation are two-fold. Firstly, to be able to make decisions on conservation management a detailed checklist for each hill is necessary and secondly, because each hill harbours only a fraction of the flora, a network of protected hills is required to capture the maximum biodiversity of the limestone flora.

Among the 267 species recorded from Batu Caves, 16 taxa $(6 \%)$ are of conservation importance, either because they are local endemics that are restricted to just Batu Caves (4 species) or within $15 \mathrm{~km}$ of Batu Caves (3

TABLE 1. Number of taxa on Batu Caves compared with the total Peninsular Malaysia limestone flora.

\begin{tabular}{llll}
\hline & Total No. Species & $\begin{array}{l}\text { No. and percentage of endemic } \\
\text { species }\end{array}$ & $\begin{array}{l}\text { No. and percentage of obligate (R) and characteristic (U) } \\
\text { calciphile species }\end{array}$ \\
\hline Batu Caves & 269 & $51(19 \%)$ & $80(30 \%)$ \\
Limestone Flora & 1,216 & $261(21.4 \%)$ & $335(27.5 \%)$ \\
\hline
\end{tabular}

${ }^{1}$ Data from Chin 1977. 
restricted to limestone and 4 on both limestone and nonlimestone substrates) and 6 are widespread elsewhere but in Peninsular Malaysia are known only from Batu Caves.

Because Ridley was collecting when the flora of Peninsular Malaysia was just beginning to be known, many new species were described from Batu Caves (Table 2). From the scientific point of view, Batu Caves is important as a living museum where scientists are able to obtain living material of the authentic specimens from the type locality, for example, for DNA analysis, breeding and other investigations.

Although the Batu Caves tower karst formation has remained largely intact in spite of quarrying activity, the surrounding area has completely changed from pristine forest when Kelsall and Ridley made their collections, to the establishment of plantations that in turn were replaced by residential and industrial buildings and expanding infrastructure associated with the Sri Subramaniaswamy Temple. While the summit and flanks are largely undisturbed, the habitats around the base and associated with the Temple Cave are under severe pressure. This puts at risk populations of sensitive species that require moist shaded conditions, such as Argostemma inaequilaterum, which used to grow at the cliff base near the Art Gallery Caves; Impatiens ridleyi that only grows around the mouth of the Temple Cave where water constantly drips down; and Epithema parvibracteatum and Monophyllaea hirticalyx that grow on the rock scree below the skylight at the back of the Temple Cave. The latter species has not been seen for some years. With the removal of tree cover, aggressive invasive species both native, e.g. species of Macaranga and Mallotus (Euphorbiaceae) and of alien origin, e.g., Piper aduncum L. and Chromolena odoratum (L.) R.M. King \& H. Rob. form thickets that smother the native flora. Of particular concern is Chromolena that in dry weather becomes a fire risk. Recently human activity resulted in vegetation fires on one face of the tower karst.

Active conservation management is required to protect these habitats from disturbance that not only drastically changes the microclimate but allows the invasion of these aggressive alien species. To protect the limestone flora there is an urgent need to clearly fence off the Public Recreation Area to prevent further intrusions.

\section{Conclusion}

Batu Caves is one of the iconic tower karsts in Peninsular Malaysia not only for its dominance of the landscape, but also for its Temple Cave that attracts hundreds of thousands of devotees. Scientifically it is important for its biodiverse flora, fauna and for its caves. Its flora is important in being species rich, including a high proportion of endemic and obligate and characteristic calciphiles, including taxa that are known only from Batu Caves, besides its historic importance as a type locality.

In view of the pressure on land from its proximity to the capital, Kuala Lumpur, there is an urgent need to enforce its legal protection status, to make clear the boundary of the Public Recreation Area, and to provide a buffer zone, preferably of tree cover, to protect the sensitive habitats at the foot of the cliffs and to provide a barrier to fire.

ACKNOWLEDGEMENTS: This study was carried out as part of the Flora of Peninsular Malaysia Project funded by the Ministry of Science, Technology and Innovation through the National Council for Scientific Research and Development under Project No. 01-04-01-000 Khas 2 entitled "Safeguarding the Forest Plant Diversity of Peninsular Malaysia" and the $10^{\text {th }}$ Malaysia Plan Development Project entitled "Dokumentasi dan Inventori Flora Malaysia".Curators of the Kepong (KEP) and Singapore (SING) Herbaria are thanked for access to their BRAHMS databases and to the University of Malaya Herbarium (KLU) for access to accession books, and to all three herbarium for permission to examine specimens in their care.

TABLE 2. Species for which Batu Caves, Selangor, Malaysia, is the type locality.

\begin{tabular}{|c|c|}
\hline ORIGINAL NAME & CURRENT NAME IF REDUCED TO SYNONOMY \\
\hline \multicolumn{2}{|l|}{ Adenoncos parviflora } \\
\hline Alyxia selangorica King & Alyxia pilosa \\
\hline Andrachne calcarea Ridl. & Leptopus australis \\
\hline Aporuellia sumatrensis C.B.Clarke var. ridleyi C.B.Clarke & Pararuellia sumatrensis var. ridleyi \\
\hline \multicolumn{2}{|l|}{ Begonia phoeniogramma } \\
\hline Boea paniculata Ridl. & Paraboea paniculata \\
\hline Boea verticillata Ridl. & Paraboea verticillata \\
\hline \multicolumn{2}{|l|}{ Bulbophyllum flammuliferum } \\
\hline \multicolumn{2}{|l|}{ Cnesmone subpeltata } \\
\hline \multicolumn{2}{|l|}{ Epithema parvibracteatum } \\
\hline Hoya occlusa Ridl. & Hoya coriacea \\
\hline \multicolumn{2}{|l|}{ Impatiens ridleyi } \\
\hline Justicia microcarpa Ridl. & Rungia laxiflora \\
\hline \multicolumn{2}{|l|}{ Ophiorrhiza fruticosa } \\
\hline \multicolumn{2}{|l|}{ Pavetta pauciflora } \\
\hline \multicolumn{2}{|l|}{ Phyllanthus erythrocarpus } \\
\hline Pilea calcarea Ridl. & Pilea fruticosa \\
\hline Polyalthia congregata & Enicosanthum congregatum \\
\hline Polytrema aequala Ridl. & Ptyssiglottis kunthiana \\
\hline Pothos lorispatha Ridl. & Pothos leptostachyus \\
\hline \multicolumn{2}{|l|}{ Pseuderanthemum lilacinum } \\
\hline \multicolumn{2}{|l|}{ Psychotria lanceolaria } \\
\hline Rhaphidophora burkillana & \\
\hline
\end{tabular}




\section{Literature Cited}

Chin, S.C. 1977. The limestone flora of Malaya. 1. Gardens Bulletin Singapore 30(1): 165-219 (http://biodiversitylibrary.org/page/43593860).

Chin, S.C. 1979. The limestone flora of Malaya. 2. Gardens Bulletin Singapore 32(1): 64-203 (http://biodiversitylibrary.org/page/43631262).

Chin, S.C. 1983a. The limestone flora of Malaya. 3. Gardens Bulletin Singapore 35(2): 137-190 (http://biodiversitylibrary.org/page/43578905).

Chin, S.C. 1983b. The limestone flora of Malaya. 4. Gardens Bulletin Singapore 36(1): 31-91 (http://biodiversitylibrary.org/page/43650020).

Henderson, M.R. 1939. The flora of the limestone hills of the Malay Peninsula. Journal of Malay Branch of Asiatic Society 17(1): 13-87.

Kiew, R. 1991. The limestone flora; pp. 42-50, in: R. Kiew (ed.). The State of Nature Conservation in Malaysia. Kuala Lumpur: Malaysian Nature Society.

Moseley, M., T.W. Lim and T.T. Lim. 2012. Fauna reported from Batu Caves,
Selangor, Malaysia, annotated checklist and bibliography. Cave and Karst Science 39(2): 77-92.

Saw, L.G, L.S.L. Chua and N. Abdul Rahim. 2009. Malaysia National Strategy for Plant Conservation. Malaysia: Ministry of Natural Resources and the Environment \& Forest Research Institute Malaysia. $63 \mathrm{pp}$.

Wycherley, P.R. 1972. Ridley and Batu Caves. Malayan Nature Journal 25(1): 22-37.

Yussof, S. 1997. The Natural and Other Histories of Batu Caves. Kuala Lumpur: Malaysian Nature Society. $55 \mathrm{pp}$.

RECEIVED: March 2014

ACCEPTED: October 2014

Published online: December 2014

EDITORIAL RESPONSIBILITY: Paul A. Egan

APPENDix 1. Checklist of Vascular Plants from Batu Caves, Selangor, Malaysia. (E endemic in Peninsular Malaysia; ET endemic in Peninsular Malaysia and Peninsular Thailand; R obligate calciphile, U characteristic species, usually found on limestone

\begin{tabular}{|c|c|c|c|}
\hline FAMILY/ SPECIES & $\begin{array}{c}\text { ENDEMISM } \\
\text { E/TE }\end{array}$ & $\begin{array}{l}\text { CALCIPHILE } \\
\text { STATUS U/R }\end{array}$ & COLLECTOR, NUMBER \\
\hline \multicolumn{4}{|l|}{ Lycophytes } \\
\hline \multicolumn{4}{|l|}{ Selaginellaceae } \\
\hline Selaginella alutacia Spring. & & & Ridley 8150 \\
\hline Selaginella mayeri Hieron & & & Foxworthy FMS 23243; Ezzawanis FRI 52478; Schuettpelz 711 \\
\hline Selaginella padangensis Hieron & & & Ridley 8667; Lee s.n. \\
\hline Selaginella stipulata (Blume) Spring. & & & Ugul \& Bala KLU 21964; Schuellpelz 712 \\
\hline Selaginella willdenowia (Desv. ex Poir.) Baker & & & Chin 325 \\
\hline
\end{tabular}

\section{Ferns \\ Adiantaceae}

Adiantum capillus-veneris L.

Schuettpelz 705

Adiantum malesianum Ghatak.

R Ridley 8142; Ding DH 712; Sinclair SFN 40058; Ezzawanis FRI 52477

\section{Aspleniaceae}

Asplenium cheilosorum Kunze ex Mett.

Asplenium macrophyllum Sw.

Schuettpelz 707

Asplenium polyodon G.Forst.

Sinclair SFN 40056

Asplenium vittiforme Cav.

U Stone 7294

Lomariopsidaceae

Cyclopeltis crenata (Fee) C.Chr.

Chin 321

Nephrolepidaceae

Nephrolepis falciformis J.Sm.

U Strugnell FMS 14617,14622

Polypodiaceae

Microsorum membranifolium (R.Br.) Ching

$\mathrm{U}$

Pyrossia stigmosa (Sw.) Ching

Ding DH 714; Ezzawanis FRI 52481

\section{Pteridaceae}

Pteris ensiformis Burm.f.

Pteris venulosa Blume

Pteris vittata $\mathrm{L}$

\section{Schizaeaceae}

Actinostachys inopinata (Selling) Reed

Nor-Ezzawanis FRI 52480; Schuettpelz 709

\section{Sinopteridaceae}

Calciphilopteris allenae (Tryon)

Calciphilopteris ludens (Wall. ex Hook.)

Hemionitis arifolia (Burm.f.) T.Moore

Merton 4103

\section{Tectariaceae}

Heterogonium pinnatum (Copel.) Holttum

Tectaria sp.

$\mathrm{R}$

Tectaria devexa (Kunze) Copel.

Allen 2323

Schuettpelz 710

Abdul-Samat 336; Schuettpelz 713

Tectaria keckii (Luersson) C.Chr.

Wyatt-Smith KEP 85208

\section{Thelypteridacea}

Amphineuron immersum (Blume) Holttum

Amphineuron opulentum (Kaulf.) Holttum

R Wyatt-Smith KEP 85208

Pronephrium triphyllum (Sw.) Holttum

Vittariaceae

Antrophyum parvulum Blume

$\mathrm{R}$

Chin 1270; Kiew RK 1618; Saw FRI 48231

R Ridley 8135; Ding DH 709

U Strugnell FMS 17078

U

Sinclair SFN 40062; Schuettpelz 708

Schuettpelz 704, 706

R Molesworth-Allen 2356; Sinclair SFN 40052

R Molesworth-Allen 2395; Strugnell FMS 14615

Schuettpelz 714

Viane \& Noe 2325

Viane \& Noe 2328
Ridley 8644; Kiew, B.H. RK 1343; Sinclair SFN 40070 


\begin{tabular}{|c|c|c|c|}
\hline FAMILY/ SPECIES & $\begin{array}{l}\text { ENDEMISM } \\
\text { E/TE }\end{array}$ & $\begin{array}{l}\text { CALCIPHILE } \\
\text { STATUS U/R }\end{array}$ & COLLECTOR, NUMBER \\
\hline Diplazium pallidum (Blume) T. Moore & & & Ridley s.n. 1896 \\
\hline \multicolumn{4}{|l|}{ Gymnosperms } \\
\hline \multicolumn{4}{|l|}{ Podocarpaceae } \\
\hline Podocarpus neriifolius D.Don & & & Wyatt-Smith KEP 93281; Ng FRI 1633 \\
\hline Podocarpus polystachyus R.Br. ex Endl. & & $\mathrm{U}$ & Ng FRI 1634 \\
\hline \multicolumn{4}{|l|}{ Dicotyledons } \\
\hline \multicolumn{4}{|l|}{ Acanthaceae } \\
\hline Justicia uber C.B.Clarke & $\mathrm{E}$ & & Strugnell FMS 14618 \\
\hline $\begin{array}{l}\text { Pararuellia sumatrensis (C.B.Clarke) Bremek. } \\
\text { var. ridleyi (C.B.Clarke) Bremek. }\end{array}$ & $\mathrm{E}$ & $\mathrm{R}$ & Ridley 8213, Strugnell FMS 17079; Kiew FRI 48226 \\
\hline Pseuderanthemum crenulatum (Lindl.) Radlk. & & & Mohd Kasim 500 \\
\hline Pseuderanthemum lilacinum Stapf & $\mathrm{E}$ & $\mathrm{R}$ & Ridley s.n. \\
\hline Ptyssiglottis kunthiana (Nees) B.Hansen & & $\mathrm{U}$ & Ridley s.n. 1914 \\
\hline Rungia laxiflora C.B.Clarke & ET & & Ridley 8213 \\
\hline \multicolumn{4}{|l|}{ Actinidaceae } \\
\hline Sauraia leprosa Korth. & & & Ridley 8269 \\
\hline Sauraia pentapetala (Jack) Hoogland & & & Ridley s.n. 1921 \\
\hline \multicolumn{4}{|l|}{ Anacardiaceae } \\
\hline Pistacia malayana M.R. Hend. & $\mathrm{E}$ & $\mathrm{R}$ & Whitmore FRI 758 \\
\hline \multicolumn{4}{|l|}{ Annonaceae } \\
\hline Enicosanthum congregatum (King) Airy Shaw & E & & Ridley s.n. \\
\hline $\begin{array}{l}\text { Goniothalamus macrophyllus (Blume) Hook.f. } \\
\text { \& Thomson }\end{array}$ & & & Syahida-Emiza FRI 66738 \\
\hline Polyalthia brunneifolia J.Sinclair & $\mathrm{E}$ & & Whitmore FRI 757 \\
\hline $\begin{array}{l}\text { Polyalthia jenkensii (Hook.f. \& Thomson)Hook.f. } \\
\text { \& Thomson }\end{array}$ & & & Bowen 8483 \\
\hline Polyalthia obliqua Hook.f. \& Thomson & & & Whitmore 79243 \\
\hline Sageraea elliptica (A.DC.) Hook.f. \& Thomson & & & Stone 1266 \\
\hline Trivalvaria macrophylla (Blume) Miq. & & & Wyatt-Smith FMS 30781; Chin SFN 40060 \\
\hline $\begin{array}{l}\text { Uvaria grandiflora Roxb. ex Hornem. var. } \\
\text { grandiflora }\end{array}$ & & & Symington FRI 66730 \\
\hline Uvaria javana Dunal & & & Sinclair FRI 700 \\
\hline \multicolumn{4}{|l|}{ Apocynaceae } \\
\hline Alstonia scholaris (L.) R.Br. & & & Hamid FMS 6443 \\
\hline Alyxia angustifolia Ridl. & $\mathrm{E}$ & & Wyatt-Smith KEP 79250 \\
\hline Alyxia pilosa Miq. & & & Ridley 8558 \\
\hline Beaumontia murtonii Craib & & & Wyatt-Smith FRI 76343; Saw FRI 48223 \\
\hline Dischidia hirsuta (Blume) Decne. & & & Rintz RER 110 \\
\hline Gymnema sp. & & & Rintz RER 10 \\
\hline Heterostemma piperifolium King \& Gamble & $\mathrm{E}$ & $\mathrm{U}$ & Burkill SFN 2261 \\
\hline Hoya coriacea Blume & & & Ridley s.n. 1890 \\
\hline Hoya finlaysonii Wight & & & Rintz RER 107 \\
\hline $\begin{array}{l}\text { Hoya verticillata (Vahl.) G.Don var. citrina } \\
\text { (Ridl.) Veldkamp }\end{array}$ & $\mathrm{E}$ & $\mathrm{U}$ & Rintz RER 111 \\
\hline Hunteria zeylanica (Retz.) Gardn. ex Thwaites & & & Ridley 8556 \\
\hline Kopsia griffithii King \& Gamble var. griffithii & & & Ezzawanis FRI 52482 \\
\hline Mardenia ridleyi P.I.Forst. & $\mathrm{E}$ & & Rintz RER 109 \\
\hline Marsdenia tinctoria R.Br. & & $\mathrm{U}$ & Ridley s.n.; Burkill SFN 6356 \\
\hline Secamone elliptica $\mathrm{R} . \mathrm{Br}$. & & & Chin 351; Rintz RER 12, 59 \\
\hline Tabernaemontana peduncularis Wall. & & & Ridley 8555 \\
\hline Toxocarpus curtisii King \& Gamble & & & Rintz RER 112 \\
\hline Toxocarpus griffithii Decne. & & & Ridley 1897 \\
\hline Toxocarpus pauciflora M.R.Hend. & $\mathrm{E}$ & $\mathrm{R}$ & Chin 353 \\
\hline Tylophora flexuosa R.Br. & $\mathrm{E}$ & $\mathrm{R}$ & Burkill SFN 6351 \\
\hline \multicolumn{4}{|l|}{ Araliaceae } \\
\hline Schefflera oxyphylla (Miq.) R.Vig. & & & Symington KEP 32652; Whitmore FRI 15634 \\
\hline \multicolumn{4}{|l|}{ Balsaminaceae } \\
\hline Impatiens ridleyi Hook.f. & $\mathrm{E}$ & $\mathrm{R}$ & Ridley 8278; Anthonysamy SA 380; Kiew RK 4706 \\
\hline \multicolumn{4}{|l|}{ Begoniaceae } \\
\hline Begonia kingiana Irmsch. & $\mathrm{E}$ & $\mathrm{R}$ & Burkill 2263; Kiew, B.H. RK 1341 \\
\hline Begonia phoeniogramma Ridl. & $\mathrm{E}$ & & $\begin{array}{l}\text { Ridley 13430; Sinclair SFN 40067; Kiew RK 1257; Symington KEP } \\
32656\end{array}$ \\
\hline
\end{tabular}




\begin{tabular}{|c|c|c|c|}
\hline FAMILY/ SPECIES & $\begin{array}{l}\text { ENDEMISM } \\
\text { E/TE }\end{array}$ & $\begin{array}{l}\text { CALCIPHILE } \\
\text { STATUS U/R }\end{array}$ & COLLECTOR, NUMBER \\
\hline \multicolumn{4}{|l|}{ Bignoniaceae } \\
\hline Radermachera glandulosa (Blume) Miq. & & & Ridley 8537 \\
\hline \multicolumn{4}{|l|}{ Boragineaceae } \\
\hline Ehretia timorensis Decne. & & & Chin 1248 \\
\hline \multicolumn{4}{|l|}{ Capparaceae } \\
\hline Capparis pubiflora DC. & & & Burkill SFN 6369 \\
\hline \multicolumn{4}{|l|}{ Celastraceae } \\
\hline Euonymus javanicus Blume & & & Symington KEP 30800 \\
\hline Glyptopetalum quadrangulare Prain ex King & & & Chin 334 \\
\hline Loeseneriella cumingii Laws. & & & Wyatt-Smith 76342; Sinclair SFN 40054 \\
\hline Maytenus curtisii (King) Ding Hou & ET & $\mathrm{R}$ & Chin 1261 \\
\hline Salacia macrophylla Blume & & & Wyatt-Smith KEP 79149 \\
\hline \multicolumn{4}{|l|}{ Chloranthaceae } \\
\hline Chloranthus erectus (Buch.-Ham.) Verdc. & & & Chin 1666 \\
\hline \multicolumn{4}{|l|}{ Convolvulaceae } \\
\hline Argyreia kunstleri (Prain) Prain ex Ooststr. & $\mathrm{E}$ & & Ridley 8220 \\
\hline Erycibe rheedii Blume & & & Burkill SFN 6365 \\
\hline Lepistemon binectiferum (Wall.) Kuntze & & & Ridley s.n.; Stone 8976 \\
\hline \multicolumn{4}{|l|}{ Cucurbitaceae } \\
\hline Bayabusua clarkei (King) W.J. de Wilde & $\mathrm{E}$ & & Ridley 8275 \\
\hline Coccinia grandis (L.) Voight & & & Syahida-Emiza FRI 66740 \\
\hline Melothria pendula L. & & $\mathrm{R}$ & Chew FRI 51926 \\
\hline Momordica cochinchinensis (Lour.) Spreng. & & & Ridley 8277 \\
\hline Neoalsomitra clavigera (Wall.) Hutch. & & & Syahida-Emiza FRI 66732 \\
\hline $\begin{array}{l}\text { Scopellaria marginata (Blume) W.J. de Wilde } \\
\text { \& Duyfjes }\end{array}$ & & & Ridley 8280 \\
\hline \multicolumn{4}{|l|}{ Dilleniaceae } \\
\hline Dillenia excelsa (Jack) Gilg & & & Ng FRI 27201 \\
\hline \multicolumn{4}{|l|}{ Dipterocarpaceae } \\
\hline Anisoptera costata Korth. & & & Strugnell 17073 \\
\hline Hopea dryobalanoides Miq. & & & Wyatt-Smith KEP 98274 \\
\hline \multicolumn{4}{|l|}{ Ebenaceae } \\
\hline Diospyros kurzii Hiern. & & & Sinclair SFN 40055; Wyatt-Smith KEP 76341 \\
\hline \multicolumn{4}{|l|}{ Elaeocarpaceae } \\
\hline Elaeocarpus pedunculatus Wall. Ex Mast. & & & Chin 1262 \\
\hline \multicolumn{4}{|l|}{ Erythroxylaceae } \\
\hline Erythroxylum cuneatum (Miq.) Kurz & & & Ng FRI 1636 \\
\hline \multicolumn{4}{|l|}{ Euphorbiaceae } \\
\hline Bridelia tomentosa Blume & & & Stone 8978 \\
\hline Cnesmone subpeltata Ridl. & $\mathrm{E}$ & $\mathrm{R}$ & Whitmore FRI 751 \\
\hline Homalanthus populneus (Grisel.) Pax & & & Sinclair SFN 40074 \\
\hline Macaranga tanarius (L.) M.A. & & & Whitmore FRI 754 \\
\hline Mallotus dispar (Blume) M.A. & & $\mathrm{U}$ & $\begin{array}{l}\text { Strugnell FMS 14619; Symington FMS 32653; Wyatt-Smith KEP } \\
94598\end{array}$ \\
\hline Mallotus repandus (Willd.) M.A. & & & Symington FMS 30793, 32654 \\
\hline Sapium insigne (Royle) Benth. & & $\mathrm{R}$ & Ng FRI 1626 \\
\hline Trigonostemon villosus Hook.f. & & & Whitmore FRI 1752 \\
\hline \multicolumn{4}{|l|}{ Gentianaceae } \\
\hline Duplipetala pentanthera (C.B.Clarke) Thiv. & & $\mathrm{U}$ & Ridley 8218; Kiew RK 1617 \\
\hline Fagraea carnosa Jack & & $\mathrm{U}$ & Wyatt-Smith KEP 85211 \\
\hline Fagraea ceilanica Thunb. & & & Ng FRI 1627 \\
\hline \multicolumn{4}{|l|}{ Gesneriaceae } \\
\hline Cyrtandra pendula Blume & & & Kiew, B.H. RK 1344 \\
\hline Epithema parvibracteatum Hilliard \& B.L.Burtt & $\mathrm{E}$ & $\mathrm{R}$ & Ridley 8217; Siti-Munirah FRI 70502 \\
\hline Microchirita caliginosa (C.B.Clarke) Y.Z.Wang & & $\mathrm{R}$ & Anthonysamy SA 379; Sinclair SFN 40066; Symington FMS 30796 \\
\hline Monophyllaea hirticalyx Franch. & $\mathrm{E}$ & $\mathrm{R}$ & Chin 2107 \\
\hline Monophyllaea horsfieldii R.Br. & & & Anthonysamy SA 378; Sinclair SFN 40061 \\
\hline Paraboea paniculata (Ridl.) B.L.Burtt & $\mathrm{E}$ & $\mathrm{R}$ & Ridley 8226; Kelsall 1970; Wyatt-Smith KEP 79245; Yusof 02 \\
\hline Paraboea verticillata (Ridl.) B.L.Burtt & $\mathrm{E}$ & $\mathrm{R}$ & Ridley 8551; Kelsall s.n.; Ng FRI 1628; Kiew FRI 48225 \\
\hline \multicolumn{4}{|l|}{ Guttiferae } \\
\hline Garcinia murdochii Ridl. & & & Chin 1253 \\
\hline
\end{tabular}




\begin{tabular}{|c|c|c|c|}
\hline FAMILY/ SPECIES & $\begin{array}{l}\text { ENDEMISM } \\
\text { E/TE }\end{array}$ & $\begin{array}{l}\text { CALCIPHILE } \\
\text { STATUS U/R }\end{array}$ & COLLECTOR, NUMBER \\
\hline \multicolumn{4}{|l|}{ Labiatae } \\
\hline Callicarpa angustifolia King \& Gamble & & $\mathrm{R}$ & Wyatt-Smith KEP 79249; Ng FRI 1637; Saw FRI 48230 \\
\hline Clerodendrum deflexum Wall. & & & Ridley s.n.; Wyatt-Smith KEP 79247 \\
\hline \multicolumn{4}{|l|}{ Lauraceae } \\
\hline Cryptocarya griffithiana Wight & & & Chin 1246 \\
\hline Dehaasia pauciflora Blume & & & Chin 328 \\
\hline Litsea angulata Blume & & & Ridley 8505 \\
\hline \multicolumn{4}{|l|}{ Lecythidaceae } \\
\hline Barringtonia fusiformis King & & & Ridley 8284 \\
\hline \multicolumn{4}{|l|}{ Leguminosae } \\
\hline Derris trifoliata Lour. & & & Stone 8980 \\
\hline Pterolobium densiflorum Prain & & & Whitmore FRI 15635 \\
\hline \multicolumn{4}{|l|}{ Melastomataceae } \\
\hline Pogonanthera pulverulenta (Jack) Blume & & & Wyatt-Smith KEP 85210 \\
\hline \multicolumn{4}{|l|}{ Meliaceae } \\
\hline Aglaia teysmanniana (Miq.) Miq. & & & Chin 1245; Putz FRI 23689 \\
\hline Chisocheton patens Blume & & & Ridley 8609 \\
\hline Chukrasia tabularis A.Juss. & & & Wyatt-Smith FRI 76344 \\
\hline \multicolumn{4}{|l|}{ Memecylonaceae } \\
\hline Memecylon lilacinum Zoll. \& Moritzi & & & Ridley 8279 \\
\hline Memecylon ovatum Sm. & & & Chin 313 \\
\hline Memecylon scutellatum var. brevifolium & & & Ridley s.n. \\
\hline \multicolumn{4}{|l|}{ Menispermaceae } \\
\hline Cyclea laxiflora Miers & & & Whitmore FRI 759 \\
\hline \multicolumn{4}{|l|}{ Moraceae } \\
\hline Ficus calcicola Corner & & $\mathrm{R}$ & Whitmore FRI 15633 \\
\hline Ficus callophylla Blume & & & Ng FRI 1635 \\
\hline Ficus hispida L.f. & & & Symington FMS 30790 \\
\hline Ficus lepicarpa Blume & & & Ridley 8185 \\
\hline Ficus punctata Thunb. & & & Ridley 8501 \\
\hline Ficus sagittata Vahl. & & & Ridley 8524, 13374 \\
\hline Ficus schwarzii Koord. & & & Ridley 8188 \\
\hline Ficus subulata Blume & & & Symington FMS 30773 \\
\hline Ficus sundaica Blume & & & Ridley s.n.; Chin 1272 \\
\hline \multicolumn{4}{|l|}{ Myrsinaceae } \\
\hline Ardisia sp. Z TFM 4:273 & $\mathrm{E}$ & & Symington KEP 30799 \\
\hline \multicolumn{4}{|l|}{ Myrtaceae } \\
\hline Rhodamnia cinerea Jack & & & Chin 1255 \\
\hline $\begin{array}{l}\text { Syzygium scortechinii (King) P. Chantaranothai } \\
\text { var. cuneatum (M.R.Hend.) I.M.Turner }\end{array}$ & $\mathrm{E}$ & & Symington FMS 30791 \\
\hline Syzygium stapfianum (King) I.M.Turner & $\mathrm{E}$ & & Wyatt-Smith KEP 80267 \\
\hline \multicolumn{4}{|l|}{ Olacaceae } \\
\hline Strombosia ceylanica Gardn. & & & Ridley 8150 \\
\hline Strombosia javanica Blume & & & Ridley 8267 \\
\hline \multicolumn{4}{|l|}{ Oleaceae } \\
\hline Jasminum cordatum Ridl. & ET & $\mathrm{R}$ & Chin 359; Syahida-Emiza FRI 66737 \\
\hline Ligustrum confusum Decne. & & $\mathrm{R}$ & Ding Hou 704; Kiew RK 1615 \\
\hline \multicolumn{4}{|l|}{ Pandaceae } \\
\hline Microdesmis caseariifolia Hook.f. ex Planch. & & & Symington FMS 30783 \\
\hline \multicolumn{4}{|l|}{ Phyllanthaceae } \\
\hline $\begin{array}{l}\text { Actephila excelsa (Dalz.) M.A. var. acuminata } \\
\text { Airy Shaw }\end{array}$ & & $\mathrm{R}$ & Chin s.n. \\
\hline Glochidion obscurum (Roxb.) ex Willd.) & & & Chin 472 \\
\hline Glochidion rubrum Blume & & & Chin s.n. \\
\hline Leptopus australis (Zoll. \& Mor.) Pojarkova & & $\mathrm{R}$ & Ridley 8203 \\
\hline Phyllanthus oxyphyllus Miq. & & & Ridley 8174 \\
\hline Phyllanthus reticulatus Poir. & & & Ridley s.n. \\
\hline Sauropus androgynus (L.) Merr. & & & Syahida-Emiza FRI 66734 \\
\hline Sauropus macranthus Hassk. & & $\mathrm{R}$ & Ridley 8183, 8257 \\
\hline \multicolumn{4}{|l|}{ Piperaceae } \\
\hline Peperomia portulacoides (Lam.) A. Dietr. & & $\mathrm{R}$ & Kelsall s.n. 1891; Ridley 19446 \\
\hline Piper argyrites Ridl. & $\mathrm{E}$ & & Ridley s.n. \\
\hline
\end{tabular}




\begin{tabular}{|c|c|c|c|}
\hline FAMILY/ SPECIES & $\begin{array}{l}\text { ENDEMISM } \\
\text { E/TE }\end{array}$ & $\begin{array}{l}\text { CALCIPHILE } \\
\text { STATUS U/R }\end{array}$ & COLLECTOR, NUMBER \\
\hline Piper kurzii Ridl. & & & Ridley 8180 \\
\hline Piper mucronatum C.DC. & E & & Poore 807 \\
\hline Piper umbellatum L. & & & Symington FMS 30794 \\
\hline \multicolumn{4}{|l|}{ Rhamnaceae } \\
\hline Sageretia thea (Osbeck) M.C.Johnst. & & $\mathrm{R}$ & Whitmore FRI 15627; Ng FRI 1638 \\
\hline Ventilago oblongifolia Blume & & & Chin 344 \\
\hline Zizyphus pernettyoides Ridl. & E & $\mathrm{R}$ & Chin 343 \\
\hline \multicolumn{4}{|l|}{ Rhizophoraceae } \\
\hline Carallia brachiata (Loir.) Merr. & & & Ridley 8265 \\
\hline \multicolumn{4}{|l|}{ Rubiaceae } \\
\hline Aidia densiflora (Wall.) Masam. & & & Whitmore FRI 755, 15632 \\
\hline Argostemma diversifolium Ridl. & E & $\mathrm{R}$ & Teruya 500 \\
\hline Argostemma inaequilaterum Benn. & & $\mathrm{U}$ & Ridley 8233; Symington KEP 30789 \\
\hline Chassalia sp. & & & Chin 474 \\
\hline Mycetia malayana (Wall. Ex Ridl.) Craib & & $\mathrm{U}$ & Hamid KEP 7011 \\
\hline Ophiorrhiza discolor R.Br. & & & Ridley s.n. \\
\hline Ophiorrhiza fruticosa Ridl & E & $\mathrm{R}$ & Ridley 8237, 8274; Ng FRI 1639 \\
\hline Ophiorrhiza pallidula Ridl. & E & & Ridley s.n. \\
\hline Pavetta pauciflora Ridl. & E & $\mathrm{R}$ & Ridley s.n. 1920 \\
\hline Psychotria lanceolaria Ridl. & $\mathrm{E}$ & & Ridley s.n. \\
\hline Psychotria penangiana Hook.f. & E & & Strugnell KEP 17081 \\
\hline Tarenna adangensis (Ridl.) Ridl. & & $\mathrm{R}$ & Ding DH 708 \\
\hline Tarenna angustifolia (King) Merr. & & $\mathrm{R}$ & $\begin{array}{l}\text { Wyatt-Smith KEP 79248; Whitmore FRI 15628; Syahida-Emiza FRI } \\
66731\end{array}$ \\
\hline Tarenna sp. 16 & E & $\mathrm{R}$ & Chin 1249 \\
\hline \multicolumn{4}{|l|}{ Rutaceae } \\
\hline Clausena excavata Burm.f. & & & Chin 345 \\
\hline Glycosmis chlorosperma Spr. & & & Symington FMS 30792 \\
\hline $\begin{array}{l}\text { Glycosmis trichanthera Guillaumin var. trichan- } \\
\text { thera }\end{array}$ & & $\mathrm{R}$ & Wyatt-Smith KEP 85207; Stone 8977 \\
\hline Paramignya scandens (Griff.) Craib & E & & Stone 8981 \\
\hline \multicolumn{4}{|l|}{ Salicaceae } \\
\hline Osmelia maingayi King & & & Ridley 8593 \\
\hline Scolopia spinosa (Roxb.) Warb. & & & Chin 354 \\
\hline \multicolumn{4}{|l|}{ Sapindaceae } \\
\hline Allophylus cobbe (L.) Raeusch. & & & Ridley s.n. \\
\hline $\begin{array}{l}\text { Dimocarpus longan Lour. subsp. malesianus } \\
\text { Leenh. }\end{array}$ & & $\mathrm{R}$ & Curtis 3773 \\
\hline \multicolumn{4}{|l|}{ Sapotaceae } \\
\hline Pouteria obovata (R.Br.) Baehni & & & Ng FRI 1623 \\
\hline \multicolumn{4}{|l|}{ Solanaceae } \\
\hline Lycianthes biflora (Lour.) Bitter & & $\mathrm{U}$ & Wyatt-Smith KEP 79150 \\
\hline Solanum erianthum D.Don & & & Merton 4105 \\
\hline \multicolumn{4}{|l|}{ Sterculiaceae } \\
\hline Pterospermum acerifolium (L.) Willd. & & & Wyatt-Smith KEP 79246; Symington FMS 30782 \\
\hline Sterculia sp. & & & Chin 1263 \\
\hline \multicolumn{4}{|l|}{ Ulmaceae } \\
\hline Celtis philippinensis Blanco & & $\mathrm{R}$ & Chin 2100 \\
\hline \multicolumn{4}{|l|}{ Urticaceae } \\
\hline Debregeasia squamata King ex Hook.f. & & $\mathrm{U}$ & Ridley s.n. 1897 \\
\hline Dendrocnide stimulans (L.f.) Chew & & & Ridley 8527 \\
\hline Elatostema curtisii (Ridl.) H.Schrot. & $\mathrm{E}$ & & Ridley 4717, 8196 \\
\hline Elatostema latifolium (Blume) H.Schrot. & & & Ridley 8200 \\
\hline Elatostema repens (Lour.) Hallier f. & & & Ridley 8186 \\
\hline Nothocnide mollissima (Blume) Chew & & & Ridley s.n. 1920 \\
\hline Oreocnide rubescens (Blume) Miq. & & & Ridley 8242; Sinclair SFN 40069; Omar FMS 7995 \\
\hline Pilea fruticosa Hook.f. & $\mathrm{E}$ & $\mathrm{R}$ & Ridley 8526; Ding DH 720 \\
\hline Poikilospermum cordifolium (Barg.-Petr.) Merr. & & & Mohd Nur 8962 \\
\hline \multicolumn{4}{|l|}{ Violaceae } \\
\hline Rinorea horneri (Korth.) Kuntze & & & Chin 338 \\
\hline \multicolumn{4}{|l|}{ Vitaceae } \\
\hline Cayratia wrayi (King) Gagnep. & & & ?Ridley \\
\hline Cissus hastata Miq. & & & Whitmore FRI 15629 \\
\hline
\end{tabular}




\begin{tabular}{|c|c|c|c|}
\hline FAMILY/ SPECIES & $\begin{array}{c}\text { ENDEMISM } \\
\text { E/TE }\end{array}$ & $\begin{array}{l}\text { CALCIPHILE } \\
\text { STATUS U/R }\end{array}$ & COLLECTOR, NUMBER \\
\hline Cissus javana DC. & & $\mathrm{R}$ & Chin 1755 \\
\hline Cissus nodosa Blume & & & Ng FRI 1631; Ding DH 711 \\
\hline Leea indica (Burm.f.) Merr. & & & Chin 476 \\
\hline Leea saxatilis Ridl. & $\mathrm{E}$ & $\mathrm{U}$ & Ridley 305, Merton 4098 \\
\hline $\begin{array}{l}\text { Tetrastigma leucostaphylum (Dennst.) Alston } \\
\text { ex Mabb. }\end{array}$ & & & Ridley \\
\hline $\begin{array}{l}\text { Tetrastigma pedunculare (Wall. ex Lawson) } \\
\text { Planch. }\end{array}$ & & & Chin 789 \\
\hline Tetrastigma scortechinii (King) Gagnep. & $\mathrm{E}$ & & ?Curtis \\
\hline \multicolumn{4}{|l|}{ Araceae } \\
\hline Alocasia inornata Hallier f. & & & Ridley 8168; Herscovitch 940101; Hay 9057 \\
\hline Alocasia longiloba 'lowii' & & $\mathrm{R}$ & Ridley s.n.; Sinclair 40071 \\
\hline Apoballis mutata (Hook.f) S.Y.Wong \& P.C.Boyce & & & Ridley s.n. \\
\hline Homalomena griffithii (Schott.) Hook.f. & & & Nicolson 1155 \\
\hline Homalomena humilis (Jack) Hook.f. & & & Ridley s.n. 1889 \\
\hline Pothos leptostachyus Schott. & & $\mathrm{R}$ & Ridley s.n. \\
\hline Rhaphidophora burkilliana Ridl. & $\mathrm{E}$ & $\mathrm{R}$ & Mohd. Nur 8965 \\
\hline Rhaphidophora montana (Blume) Schott. & & $\mathrm{R}$ & Ridley s.n. 1889 \\
\hline $\begin{array}{l}\text { Schismatoglottis calyptrata (Roxb.) Zoll. \& } \\
\text { Moritzi }\end{array}$ & & & Ridley s.n. 1889 \\
\hline Scindapsus hederaceus Miq. & & & Ridley s.n. \\
\hline Scindapsus perakensis Hook.f. & & & Ridley s.n. \\
\hline Typhonium fultum Ridl. & ET & $\mathrm{R}$ & Ridley 8165 \\
\hline \multicolumn{4}{|l|}{ Convallariaceae } \\
\hline $\begin{array}{l}\text { Peliosanthes teta Andrews } \\
\text { subsp. humilis (Andrews) Jessop }\end{array}$ & & & Sinclair SFN 40059 \\
\hline \multicolumn{4}{|l|}{ Dracaenaceae } \\
\hline Dracaena sp. & & & Saw FRI 48224 \\
\hline \multicolumn{4}{|l|}{ Gramineae } \\
\hline Dichanthium mucronulatum R.K.Jansen & $\mathrm{E}$ & $\mathrm{R}$ & Ridley 8129; Chin 1271 \\
\hline Echinochloa colona (L.) Link & & & Duistermaat FRI 51920 \\
\hline Eleusine indica (L.) Gaertn. & & & Duistermaat FRI 51922 \\
\hline Eragrostis pilosa (L.) P.Beauv. & & & Duistermaat FRI 51925 \\
\hline $\begin{array}{l}\text { Eragrostis amabilis (L.) Wight \& Arn. ex Hook. } \\
\text { \& Arn. }\end{array}$ & & & Duistermaat FRI 51924 \\
\hline Oplismenus compositus (L.) P.Beauv. & & & Duistermaat FRI 51916 \\
\hline $\begin{array}{l}\text { Sporobolus indicus (L.) R.Br. var. flaccidus } \\
\text { (Roem. \& Schult.) Veldkamp }\end{array}$ & & & Duistermaat FRI 51921 \\
\hline \multicolumn{4}{|l|}{ Orchidaceae } \\
\hline Adenoncos parviflora Ridl. & & & Kelsall s.n. 1891 \\
\hline Adenoncos sumatrana J.J.Sm. & & & Ridley 8171 \\
\hline Appendicula anceps Blume & & & Saw FRI 48228 \\
\hline Bulbophyllum flammuliferum Ridl. & $\mathrm{E}$ & $\mathrm{R}$ & Ridley s.n. \\
\hline Calanthe ceciliae Reichb.f. & & & Ridley 8486 \\
\hline Calanthe vestita Lindl. & & $\mathrm{R}$ & Ridley s.n. Dec 1896; Strugnell FMS 17077 \\
\hline Corybas calcicolus J. Dransf. \& G.Smith & $\mathrm{E}$ & $\mathrm{R}$ & Chin 355; Dransfield s.n. 1970 \\
\hline Corymborkis veratifolia Blume & & & Ridley 8128 \\
\hline Dendrobium subulatum (Blume) Lindl. & & & Ridley s.n. 1894 \\
\hline Goodyera pusilla Blume & & $\mathrm{R}$ & Symington FMS 30776 \\
\hline Grosourdya appendicula (Blume) Reichenb.f. & & $\mathrm{U}$ & Ridley s.n. 1897 \\
\hline Oberonia sinuosa Ridl. & & & Ridley s.n. \\
\hline Pennilabium angraecum (Ridl.) J.J.Sm. & $\mathrm{E}$ & & Ridley 8131 \\
\hline Pholidota imbricata Hook. & & $\mathrm{U}$ & Ridley s.n. 1897 \\
\hline Phreatia plantaginifolia (K.D.Koenig) Ormerod & & & Ong FRI 67738 \\
\hline Polystachya concreta (Jacq.) Garay \& H.R.Sweet & & & Saw FRI 48229; Ong FRI 71387 \\
\hline Pomatocalpa andamanica (Hook.f.) J.J.Sm. & & $\mathrm{R}$ & Chin 1244 \\
\hline Renantherella histriconica (Reichb.f.) Ridl. & & & Chin 1264 \\
\hline Schoenorchis micrantha Blume & & & Ridley s.n 1896 \\
\hline Taeniophyllum filiforme J.J.Sm. & & & Ridley s.n. 1896 \\
\hline Thelasis pygmaea Blume & & & Ridley s.n. 1896,8465 \\
\hline Trichoglottos retusa Blume & & $\mathrm{U}$ & Kelsall s.n. \\
\hline Ventricularia tenuicaulis (Hook.f.) Garay & & $\mathrm{U}$ & Ridley 8133 \\
\hline
\end{tabular}




\begin{tabular}{|c|c|c|c|}
\hline FAMILY/ SPECIES & $\begin{array}{c}\text { ENDEMISM } \\
\text { E/TE }\end{array}$ & $\begin{array}{l}\text { CALCIPHILE } \\
\text { STATUS U/R }\end{array}$ & COLLECTOR, NUMBER \\
\hline \multicolumn{4}{|l|}{ Palmae } \\
\hline Calamus manan Miq. & & & Loh FRI 21521 \\
\hline Calamus scipionum Lour. & & & Tahir FRI 18537 \\
\hline Iguanura wallichiana (Wall. ex Martelli) Hook.f. & & & Kiew, B.H. RK 1345 \\
\hline Maxburretia rupicola (Ridl.) Furtado & $\mathrm{E}$ & $\mathrm{R}$ & Ridley 8285; Whitmore FRI 15636; Saw FRI 48227 \\
\hline Oncospermum horridum (Griff.) Scheff. & & & Chin, S.C. obs. \\
\hline \multicolumn{4}{|l|}{ Pandanceae } \\
\hline Pandanus penangensis Ridl. & $\mathrm{E}$ & $\mathrm{U}$ & Whitmore FRI 15625 \\
\hline \multicolumn{4}{|l|}{ Zingiberaceae } \\
\hline Alpinia javanica Blume & & & Ridley s.n. 1889 \\
\hline Amomum testaceum Ridl. & & $\mathrm{U}$ & Ridley 13122 \\
\hline Etlingera littoralis (J.Koenig) Giseke & & & Ridley s.n. 1890 \\
\hline
\end{tabular}

